\title{
Study on the Surveillance System of Water Stage Used the Resonator Pressure Transducer and Wireless Connectivity
}

\author{
Bing Han and Qiang Fu ${ }^{a}$ \\ China National Institute of Standardization, 100191, Beijing, China
}

\begin{abstract}
For the sake of ameliorating the faultiness of low precision for conventional surveillance methods of water stage, and realize the goal of real time data collection, automated actions and long-distance conveying, we have designed a novel surveillance system of water stage with the resonator pressure transducer and wireless connectivity technologies. The surveillance system of water stage has come into service in a field experiment project of a certain oil and gas pipeline engineering. By analyzing and comparing the results of experiments, the system has the merits of high agility, reliability, instantaneity and accuracy, low cost, capacity of resisting disturbance, which making it ideal for use in unattended supervising of water stage for multi-spots observation based on regional scale. The surveillance system can well satisfy the actual demand of auto hydrogeological parameters monitoring for geotechnical engineering.
\end{abstract}

\section{Introduction}

Water stage is one of the fundamental hydrographic features of evidence for deluge circumstances of streamway or reservoir region, and it is also the key element of hydrology condition and the fundamental projects of hydrometry. It is vital that the parameter exact measurement for water conservancy construction, water release, flood operation and deluge forecast. Currently, the monitoring design programmes of water stage are mainly focusing on all sorts of transducer techniques, for instance, the float-type, piezo-resistive type, air-bubble type and electronical water gauge and so on [1-4]. There are more or less faultiness exists in these conventional methods of measurement above, including large power dissipation, poor degree of accuracy, weak anti-jamming, easy to burnin or abrasion, poor scalability, wiring intricacies, discommodiousness for assemblage and service. Some new non-contact water gauges, for instance, sonic wave, laser or radar, have the advantages of high-accuracy, large scale, modern techniques, but these instruments demand stringent specification and they are high-priced items, in addition, the survey angles have an influence on the range of measurement, which bring about the high rate of occurrence of failures and false alarm, so the fixed time adjustment is required [5-7].

The resonator pressure transducer is designed with a tight metal string as the sensitive element extensive used in engineering and scientific research field. The resonator pressure transducer outputs the eigentone signal of the metal string, it has a good disturbance resistance, little affected by the electromagnetic interference, faintest null shift, etc. The resonator pressure transducer is extensive applied in engineering surveying domains, including port engineering, mine, oil and gas pipeline, foundation, water conservancy [8-10], and shows its broad application and development prospects. We have designed a monitoring system with the resonator pressure transducer integrating survey accuracy, unattended operation, constancy and so on. This system is built with ZigBee, a short-distance twoway wireless communication technology, which is based on IEEE802.15.4 with $2.4 \mathrm{GHz}$ as the main frequency band. ZigBee can be embedded in all sorts of equipment by using direct sequence spread spectrum technology, provided with the locate function, and satisfy well the performance guideline of industrial computer, so it has been highly applied in long-distance measuring system based on the wireless network [11-12]. In this system, the monitoring data are transmitted to the remote supervisory terminus by means of the combined effort of ZigBee and GPRS, so the long-distance and round-the-clock monitoring of the water stage can be realized.

\section{Structure and principle}

\subsection{Principle of resonator pressure transducer}

The sensory unit of the resonator pressure transducer is a metal string with fixed-ends, and it is fabricated from wolfram steel, stainless steel or spring steel in generally. If the stress $F$ was applied, there will be tensile force generation in the metal string, and distrinct eigentones will be engendered consistent with diverse tensions and metal string lengths. By measuring the diversification of eigentones of the metal string, we can ascertain the diversification of internal tension, and then obtain the

a Corresponding author: fuqiang@cnis.gov.cn 
strain of the metal string. The eigentone $f$ of the metal string can be written as:

$$
f=\frac{1}{2} \sqrt{\frac{F}{m_{0} l_{0}}}
$$

Based on the mechanics of materials, we have:

$$
F=\sigma S_{0}=E_{0} S_{0} \varepsilon
$$

Where, $l_{0}, m_{0}$ and $S_{0}$ indicate the length, quality and intersecting surface of the metal string, while $E_{0}, \varepsilon$ and $\sigma$ indicate elasticity modulus, strain and stress of the metal string.

Combining expressions (1) and (2), we can evaluate the strain of resonator pressure transducer, as below

$$
\varepsilon=\frac{4 m_{0} l_{0}}{E_{0} S_{0}} f^{2}
$$

\subsection{Water stage gauge}

As the figure 1 shows, the body of water stage gauge is a seal chamber with a built-in thin thickness tube. The three resonator pressure transducers, Vib-W1, Vib-W2 and Vib$\mathrm{W} 3$, have qualified temperature compensation function, which are fixed to the intersecting surface of tube with the alternation of 90 degrees. The resonator pressure transducers are linked in sequence by melting welding and drawn into the connecting box with coaxial cable.

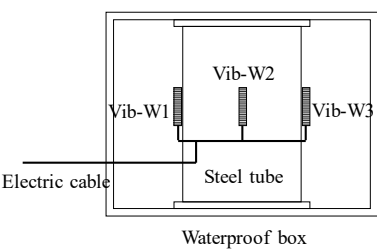

(a) Vertical section

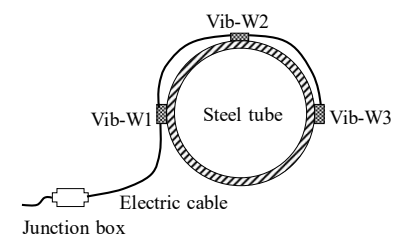

(b) Section plan
Figure 1. The assumption diagram of water stage gauge.

On the basis of fluid statics, we can evaluate $\mathrm{P}$, the hydrostatic pressure, as follow

$$
\mathrm{P}=P_{0}+\gamma h
$$

Where $P_{0}$ is barometric pressure, $\gamma$ is volume-weight of water, $h$ is depth. If the water stage change, $P$ will produce physical change, the diversification of $P$ will have an impact on the seal chamber, and the strain of thin thickness tube will come into being. According to the elastomechanics principle, $P$ can be written as follow

$$
\mathrm{P}=\mathrm{E} \varepsilon_{m}
$$

$\varepsilon_{m}$ is the average strain on intersecting surface of tube, we can evaluate the value of $\varepsilon_{m}$ by means of the three-point method, which as the Figure 2 shows.

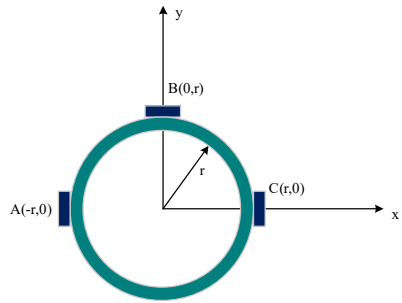

Figure 2. The three-point method.

The axial strain on any intersecting surface of tube can be evaluated by the following expression,

$$
m x+n y+p z=1
$$

$x$ and $y$ are the coordinates of any point in intersecting surface, $z$ is the axial strain of point $(x, y), m, n$ and $p$ are random constants. According to three given strains, A, B and $\mathrm{C}$, we can evaluate the strain $z$. The computational formula as below

$$
\mathrm{z}=\frac{A+C}{2}+\left(\frac{C-A}{2}\right)\left(\frac{x}{r}\right)-\left(\frac{A+C-2 B}{2}\right)\left(\frac{y}{r}\right)
$$

In consideration of the symmetrical characteristic of strain on $x$ axis, we have taken the derivative of $x$ with regard to expression (7) to obtain the maximum or minimum strain. If we make the value is equal to zero, the coordinates of $x$ and $y$ relevant to the maximum or minimum strain can be evaluated. Take the derivative of expression, which can be writen as

$$
\begin{gathered}
\frac{\mathrm{x}}{r}=\frac{C-A}{\sqrt{2 A^{2}+2 C^{2}+4 B^{2}-4 A B-4 B C}} \\
\frac{\mathrm{y}}{r}= \pm \sqrt{1-\left(\frac{x}{r}\right)^{2}}
\end{gathered}
$$

Based on the expressions (7), (8) and (9), the maximum and minimum strain on monitoring intersecting surface of tube, $z_{\max }$ and $z_{\min }$, can be evaluated, and then the mean strain on monitoring intersecting surface, $\varepsilon_{m}$ can be otained with the expression, as below

$$
\varepsilon_{m}=\frac{z_{\max }+z_{\min }}{2}
$$

After substitution of the expressions (7), (8), (9) into $(10), \varepsilon_{m}$ can be written as follow

$$
\varepsilon_{m}=(A+C-2 B) \sqrt{\frac{A^{2}+4 B^{2}+C^{2}+2 A C-4 A B-4 B}{2 A^{2}+4 B^{2}+2 C^{2}-4 A B-4 B C}}
$$

Combining the expressions (4), (5) and (11), we can evaluate water stage $h$, as below

$$
\mathrm{h}=\frac{(A+C-2 B) E \sqrt{\frac{A^{2}+4 B^{2}+C^{2}+2 A C-4 A B-4 B C}{2 A^{2}+4 B^{2}+2 C^{2}-4 A B-4 B C}}-P_{0}}{\gamma}
$$

On the basis of Eqs. (12), $h$ can be acquired with the monitoring system by applying the resonator pressure transducers discussed above. 


\section{The surveillance system}

\subsection{Construction}

The first part of surveillance system is ZigBee wireless transducer network, which is comprised of mulriple transducer nodes loaded with resonator pressure transducer in the surveillance field. All of these transducer nodes are coupled together to coordinator with star-shape topologic architecture. These transducer nodes are responsible for collecting and transmitting surveillance data to the coordinator node in the form of wireless. The network coordinator node is coupled to a GPRS unit, and the EIA-RS-232C standard protocol is applied to communication between network nodes and GPRS unit.

The second part of surveillance system is GPRS network, which is responsible for sending surveillance data to remote supervisory terminus, where the surveillance data are converged in the coordinator node by means of GPRS unit.

The third part of surveillance system is supervisory terminus. The server-centric is coupled to the GPRS unit of upper monitor with EIA-RS-232C standard protocol, and its main function is to store, handle and analyze data. In case of the dangerous levels of water stage is achieved, a warning sign will be presented by the server-centric.

Figure 3 is the abridged general view of the surveillance system.

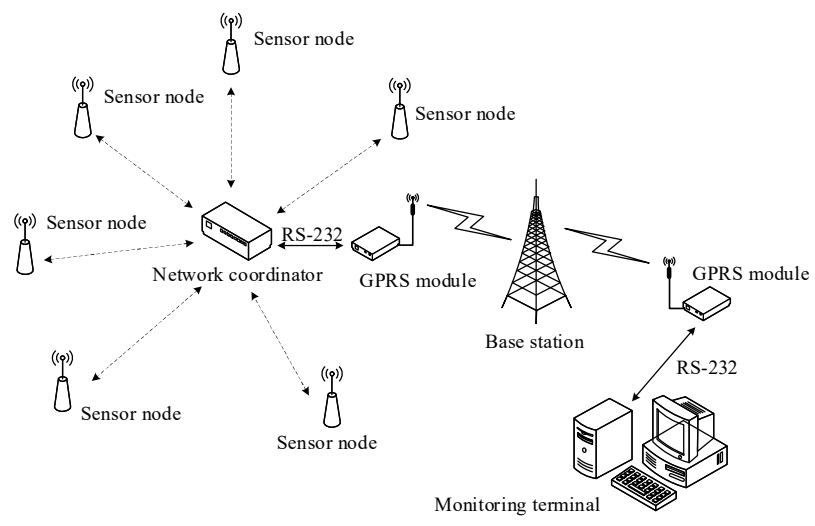

Figure 3. The abridged general view of the surveillance system.

\subsection{Hardware conditions}

A ZigBee transducer network is comprised of two components: a coordinator node and several transducer nodes, the latter is constituted of resonator pressure transducer, singlechip unit and RF wireless unit. When the surveillance data has been received, they will be transformed into the binary numerical signals and transmitted to the singlechip unit. The system power gain supply from storage batteries. The coordinator node is composed of three components: singlechip unit, RF wireless unit and GPRS unit, and its essential functions contain collecting data, dealing with data, communicate with the slave machine and carrying out the control commands. Figure 4 is the abridged general view of hardware condition of surveillance system.

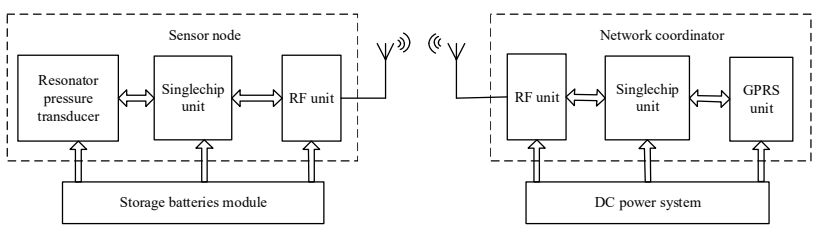

Figure 4. The bridged general view of hardware conditions.

\subsection{Software conditions}

We can develop the communication procedure on the basis of the TCP/ IP protocol of ZigBee.The software conditions consist of network entry for transducer nodes, data collection, storage and delivery; Ad Hoc network for coordinator node, monitoring, communication, data receiving and delivering with GPRS unit. When the surveillance system start operating, the coordinator node will receive an instruction from the controlling terminal to inform the wireless network coordinator to work, and all the transducer nodes will perform hardware initialization after receiving an instruction from coordinator node, meanwhile, the monitoring message from the upper monitor will be dispatched to the transducer nodes through ZigBee wireless network. The above a series of instructions make the transducer nodes to invoke the functional element program of water stage surveillance. All of the transducer nodes are the implementation component of data collection and controlling device with regard to the surveillance system, and their main functions are to accept data from network coordinator and changing the strain values of transducers into water stage by data processing.

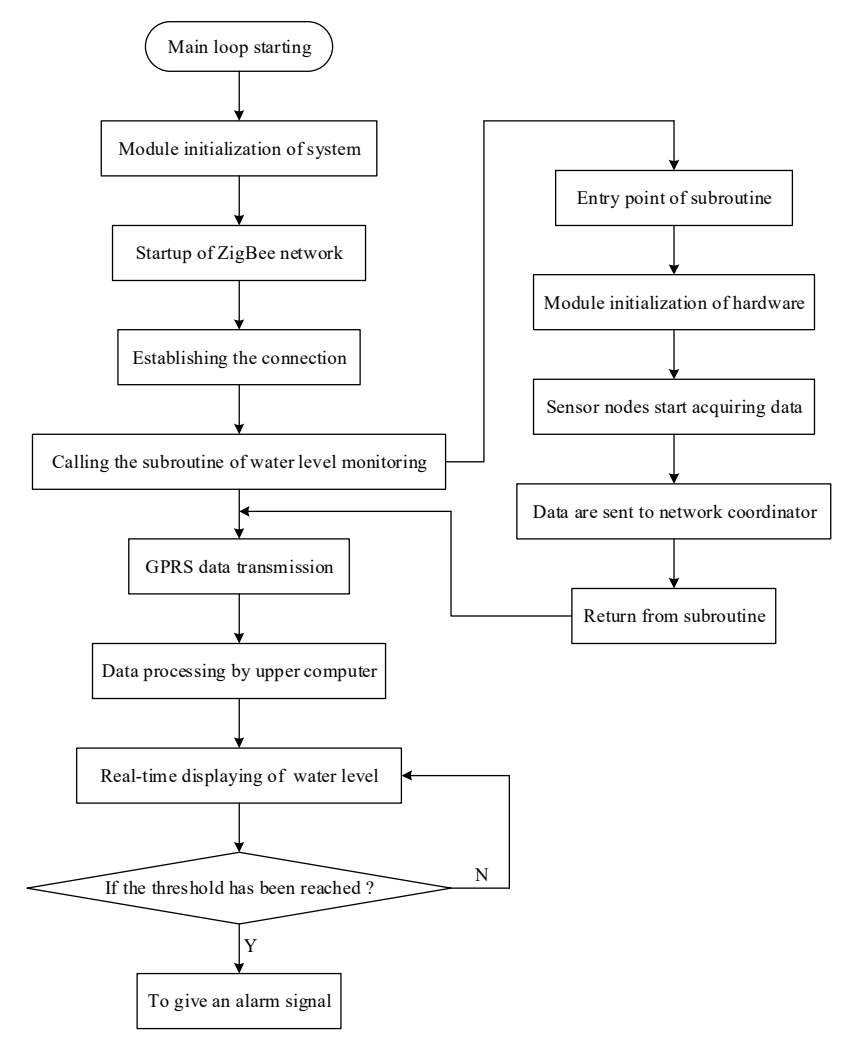

Figure 5. The bridged general view of software conditions. 
The monitoring data collected by transducer nodes are sent to the upper monitor by the coordinator node. By data procession, we can obtain the real-time curve of water stage, if the dangerous levels of water stage is achieved, a warning sign will be presented by the server-centric.

Figure 5 presents the bridged general view of software conditions of surveillance system.

\subsection{ZigBee network}

The wireless network node of ZigBee consists of radio frequency transponder and singlechip. CC2431 is a sort of $2.4 \mathrm{GHz}$ radio frequency transponder with built-in the positioning engine technology, which is applicable to all kinds of ZigBee wireless network nodes, such as coordinator, router and other end instrument. The work pattern of CC2431 is set as four serial peripheral interface bus, which can achieve caching data and read-write of temporary storage. The Implementation of setting lunch or receive buffer, dominating the estimation of clean-up channel and importing the timepiece or timing information functions can be achieved by means of the pins FIFOP, SFD, FIFO, and CCA. It is obliged to keep consistently the pin CSn low-level outputs during data conveying.

The surveillance system adopts the ultra-low power MSP430F2274 MCU as control center, which is mixed signal controller, and it has characterized by normal operation pattern and four low power consumption operation patterns. It is easy to achieve changing-over for these patterns MSP430F2274, which is characterized by high level of integration, such as the accurate comparing unit in chip, multicentre A/D conversion, bevel edge A/D conversion. Figure 6 is the schematic diagram of interface circuit.

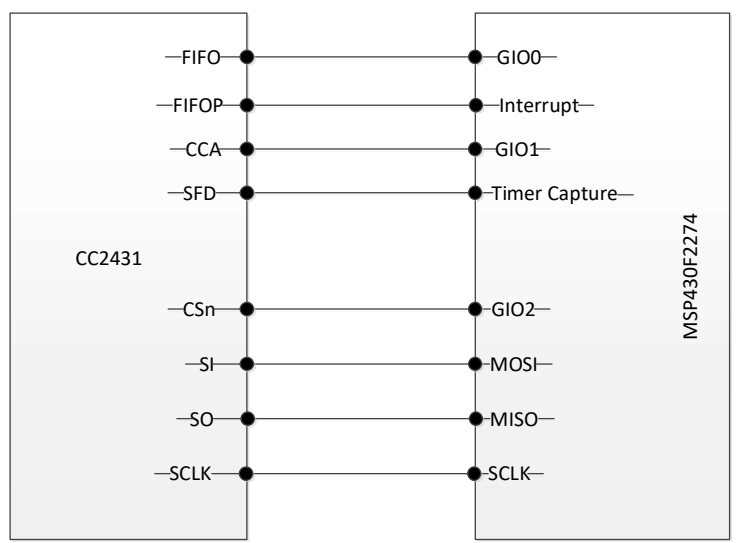

Figure 6. The schematic diagram of interface circuit.

\section{Field examples}

We have conducted the field experiments in Jiudao River Valley of Wanjialing Town, Wafangdian Country, Dalian City, Liaoning Province, in northeast China. For the sake of confirmation of this surveillance system, we have adopted two types of measurement technologies for water stage, the resonator pressure transducer and float-type sensor.
Jiudao River is a representative ephemeral stream, and there is a great disparity of water level during the drought period and high-water season. As is shown in Figure 7, we have presented the measurement data of water stage for continuous 72 hours during rainfall. The red line represents the water stage based on resonator pressure transducer, and the green line represents the water stage based on the float-type sensor, which suggests that the measurement results obtained from two kinds monitoring methods have matched each other. The trend of water stage change is essentially in agreement the precipitation data from weather station.

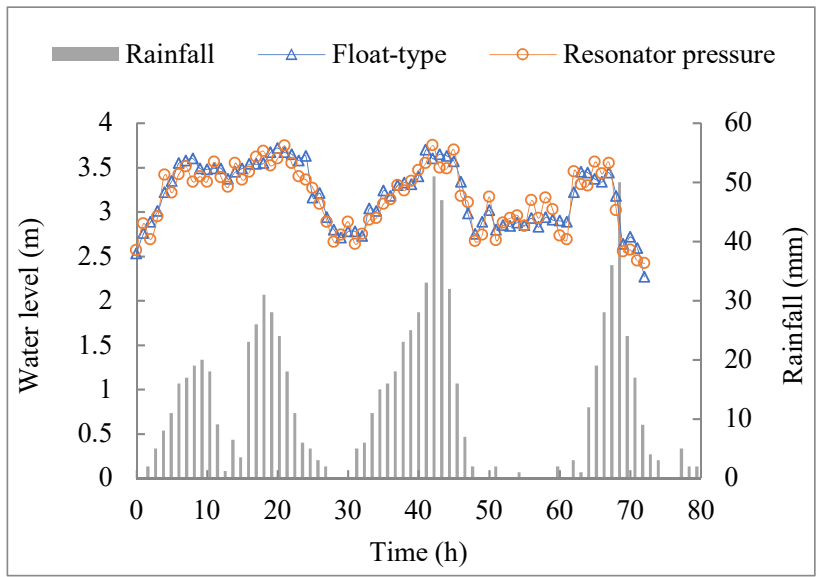

Figure 7. The measurement results of water stage.

\section{Conclusions}

In this paper, we have designed a novel surveillance system of water stage with resonator pressure transducer technology, and it is provided with high-precision, antijamming from electro-magnetic disturbance, corrosion preventive. To meet the needs of automatic data acquisition and round-the-clock monitoring, the ZigBee wireless technology has been used to construct a monitoring network, where all the sensor nodes in scene are connected to each other. The GPRS unit is applied to realize the data transmission between central node of ZigBee and server-centric. Finally, a field experiment has been conducted in Jiudao River Valley in northeast China on the basis of the surveillance system with resonator pressure transducer technology, and the test results tell us that the system features simple structure, high accuracy, good performance for ad hoc network, low power consumption and offers practical applicable value.

\section{References}

1. Y. Hou, X. H. Yu, J. Zhang, Z. Y. Meng, Y. Guo, L. Ma, Research and Application on New Float Type Water Level Gauge. Water Resources Informatization, 5, 36-39 (2012).

2. W. H. Li, C. H. Che, Study on the Problems of Groundwater Level Monitoring in Deep Foundation Pit Engineering in Urban Area. Exploration Engineering: Rock \& Soil Drilling and Tunneling, 40(6), 70-72 (2013). 
3. B. Lan, Z. Wu, G. L. Xu, Application of unsteady bubble level gauge in hydrological telemetry. Meteorological, Hydrological and Marine Instruments, 29(1), 67-70 (2012).

4. X. T. Sheng, X. Q. Gu, P. Z. Ding, S. L. Li, G. S. Zhu, M. Yan, Horizontal Drainage Holes in Bank Slope: Insitu Test and Groundwater Monitoring, Journal of Yangtze River Scientific Research Institute, 34(2), 9498 (2017)

5. Z. G. Gai, J. Zhao, L. Yang, X. Cui, C. J. Lu, S. B. Chu, J. X. Yang, B. Zhao, Development of a new laser intelligent water level measuring system, Journal of Optoelectronics Laser, 24(3), 569-572 (2013).

6. C. L. Dai, W. P. Sun, W. H. Tang, Application of the Integrated Acoustic Wave Type Water Level and Rainfall Telemetry Station in Small Reservoirs. Hydropower and New Energy, 146, 51-53 (2016).

7. L. Xu, Y. L. Wang, X. J. Hua, Research on application of radar level meter in automatic water regime monitoring system. Yangtze River, 45(2), 74-77 (2014).
8. W. Xu, Q. Zhang, W. Zhou, Development and Application of Metal string Sensor Monitoring System Based on Wireless Network with Micropower Consumption. Journal of Yangtze River Scientific Research Institute, 32(10), 134-138 (2015).

9. Z. H. Xu, J. Wang, Design of deep foundation pit monitoring system based on metal string sensor. Transducer and Microsystem Technologies, 34(7), 79-84 (2015).

10. L. Chen, Analysis of Influence of Temperature on Stress Testing of Concrete Bridge Using Metal string Sensors. Transportation Science \& Technology, 2: 2830 (2015).

11. Y. Fan, Y. Yu, Y. Y. Li, Y. X. Ding, Research on Remote Monitoring System Based on ZigBee Wireless Sensor Network. Research and Exploration in Laboratory, 35(1), 80-84 (2016).

12. Y. Q. Jiang, Z. G. Gu, W. W. Shi, Multifunctional wireless measurement and control system based on ZigBee and GPRS. Journal of Mechanical \& Electrical Engineering, 31(6), 814-818 (2014). 